\title{
Hermitian Symmetry on a Hilbert Space-Its Applications to Some Asymmetric Phenomena
}

\author{
Naohito Chino \\ Department of Psychology, Faculty of Psychological and Physical Science, Aichi Gakuin Universty, Aichi, Japan \\ Correspondence to: Naohito Chino, chino@dpc.agu.ac.jp \\ Keywords: Hermitian Symmetry, Hilbert Space, Asymmetric Similarity, Asymmetric MDS \\ Received: March 11, $2020 \quad$ Accepted: April 26, $2020 \quad$ Published: April 29, 2020 \\ Copyright (c) 2020 by author(s) and Scientific Research Publishing Inc. \\ This work is licensed under the Creative Commons Attribution International License (CC BY 4.0). \\ http://creativecommons.org/licenses/by/4.0/ \\ (c) (i) Open Access
}

\section{ABSTRACT}

The purpose of this paper is threefold. One is to revisit the Hermitian form model (HFM) with Hermitian symmetry proposed by Chino and Shiraiwa (1993), which uncovers the latent Hilbert space structure or the indefinite metric space structure, given the asymmetric similarity matrix (ASM) among objects, and another is to explain how to interpret the configuration of objects embedded in these spaces. The final goal of this paper is to show what kinds of information are obtained by applying HFM to empirical and hypothetical ASMs. Results of applications of HFM to two empirical ASMs suggest that some possible asymmetric structures among objects exist, which might not have been found empirically. The result of application of the HFM to a hypothetical ASM uncovers interesting latent space structures among objects.

\section{INTRODUCTION}

Increasing attention has been paid to mutual interactions among objects, especially in the medical and biological literature recently (e.g., [1-6]). Interactions among objects in the literature are generally asymmetric in contrast to those in the physical world. For example, Imai and Guarente report biosynthetic pathways of nicotinamide adenine dinucleotide $\left(\mathrm{NAD}^{+}\right)$[1]. As can be seen in a subsequent section, such a pathway can be converted into an asymmetric matrix, elements of which are binary, since it can be expressed as a certain directed graph (digraph) in graph theory (e.g., $[7,8])$. In quorum sensing, some bacteria secrete a signaling molecule called autoinducer to their host environment and detect their number, and if it reaches a threshold they respond again to the environment by secreting some special substance (e.g., $[2,5])$. Reference [4] studies the midbrain dopamine system, in which dopamine neurons in the ventral tegmental area (VTA) are thought to compute reward prediction error (RPE) (e.g., [9]). They demonstrate that even simple arithmetic computations such as RPE are not localized in specific brain areas but, rather, are distributed across multiple nodes in a global brain network. Such a network might also be described by a complicated digraph whose corresponding weight matrix is generally asymmetric. 
Asymmetry can be observed in the other literature such as in the social and behavioral sciences (e.g., [10]). Reference [11] summarizes various asymmetric phenomena in the various disciplines of science and discusses the meaning of asymmetry. Reference [12] analyzes the trade imbalance between 10 nations and depicts their asymmetric relations graphically using one of the asymmetric multidimensional scaling (asymmetric MDS) methods in psychometrics developed by the author.

Asymmetry is sometimes not observed but is hypothesized in some literature. For example, Sato, Akiyama, and Farmer assume a hypothetical asymmetric matrix in investigating the problem of learning to play the game of rock-paper-scissors based on the theory of games and the notion of evolutionally stable strategy (ESS) [3], which was introduced by [13].

In contrast, three of the four fundamental forces in the natural world, i.e., gravitation, electromagnetism, and strong interaction are all symmetric. One of the reasons why interactions among these objects in the medical and biological sciences as well as the social and behavioral sciences are generally asymmetric might be that living organisms have intelligence to some degree in the sense of [14], even if they have no neurons (e.g., [6]), and as a result they exert a force on their environment. Wechsler defines the intelligence as the aggregate or global capacity of the individual to act purposefully, to think rationally and to deal effectively with his or her environment.

Generally, it will be natural and appropriate to discuss two aspects if we consider mutual interactions among objects. One is the space in which objects interact with one another, and the other the dynamics by which objects interact. From the dynamical system point of view, the former is the state space, and the latter the equation of motion of objects in that space.

In this paper, we shall restrict our attention to the former and discuss the space in which objects are embedded. For example, in the special relativity theory a special quadratic form, $\mathrm{d} s^{2}=\sum_{i, j} g_{i j} \mathrm{~d} x_{i} \mathrm{~d} x_{j}$, holds for definite differentials, $\mathrm{d} x_{1}, \mathrm{~d} x_{2}, \mathrm{~d} x_{3}, \mathrm{~d} x_{4}$, of the four-dimensional space-time coordinates of any chosen system of reference, where $x_{1}, x_{2}, x_{3}$ are the spatial coordinates and $x_{4}$ is the corresponding timecoordinate measured by some suitable measuring clock (e.g., [15]). Moreover, the matrix $\boldsymbol{g}_{i j}\left(=\left[g_{i j}\right]\right)$ is a special diagonal matrix whose diagonal elements are $(-1,-1,-1,+1)$, which is called a metric tensor. The state space described by this tensor has a Minkowski metric. In the general relativity theory, this tensor is no longer constant, but a function of space and time. In this case, the state space is a Riemannian space with a Riemannian metric which is symmetric. In quantum mechanics, the Born rule is one of the key principles, and it states that if an observable corresponding to a Hermitian operator (or sometimes called a self-adjoint operator), say, $\hat{\boldsymbol{O}}$, with discrete spectrum is measured in a system with normalized wave function, the kets $|\psi\rangle$, in a Hilbert space [16], then the measured result is one of the eigenvalues $\rho$ of $\hat{\boldsymbol{O}}$, where $|\cdot\rangle$ is the ket vector. $\hat{\boldsymbol{O}}$ is called the density matrix operator [17], and if we choose the kets $\left|\psi_{k}\right\rangle$ to be the eigenvectors (or eigenkets) of $\hat{\boldsymbol{O}}$ with eigenvalues $\lambda_{k}$, we can write $\hat{\boldsymbol{O}}=\sum_{k} \rho_{k}\left|\psi_{k}\right\rangle\left\langle\psi_{k}\right|$, where $\langle\cdot|$ is the bra vector.

Then, what is an appropriate mathematical space in which objects are embedded with asymmetric relationships among them? According to [18], this space is nothing but a Hilbert space with discrete spectrums. The organization of this paper is as follows: In Section 2, we shall revisit briefly the Hermitian Form Model (abbreviated as HFM), which was proposed by Chino and Shiraiwa [18]. In this section, we see that our HFM has a property of Hermitian symmetry. In Section 3, we shall discuss briefly the similarities and differences in Hilbert space used in quantum mechanics and psychometrics, especially in HFM. In order to do so, we shall restrict our attention to finite-dimensional Hilbert space, although in general Hilbert space is defined to be infinite-dimensional in quantum mechanics. Finally, we shall apply the HFM to several asymmetric matrices among objects obtained in the medical and biological sciences as well as the social and behavioral sciences.

\section{REVISIT OF HFM}

\subsection{Brief Derivation of HFM}

In this section we shall briefly revisit HFM according to [18] and [19]. The data matrix to which we apply is an $N$ by $N$ asymmetric similarity matrix (abbreviated hereafter as $A S M$ ), $\boldsymbol{S}=\left[s_{j k}\right]$, where $s_{j k}$ 
denotes the magnitude of similarity from object $j$ to object $k$. The $s_{j k}$ must be measured at the ratio level in HFM. The ratio level means in psychometrics that $s_{j k}$ must be measured by some scale with equal adjacent intervals and absolute zero. In HFM, we first decompose $S$ into the symmetric part and the skewsymmetric part as

$$
S=S_{s}+S_{s k}
$$

where $\boldsymbol{S}_{s}=\left[\frac{s_{j k}+s_{k j}}{2}\right]$ and $\boldsymbol{S}_{s k}=\left[\frac{s_{j k}-s_{k j}}{2}\right]$. Then we construct a Hermitian matrix as follows:

$$
\boldsymbol{H}=\boldsymbol{S}_{s}+i \boldsymbol{S}_{\text {sk }},
$$

where $i^{2}=-1$. We shall call the one-to-one correspondence between $S$ in Equation (1) and $H$ in Equation (2) the Chino-Shiraiwa rule. This rule means that we let any measured or hypothesized ASM correspond to a Hermitian matrix which cannot be observed. In this sense, our rule might be contrasted with the Born rule in quantum physics [16]. Moreover, the Chino-Shiraiwa rule enables us to deduce a holistic mathematical space structure in which objects are embedded as will be shown below.

In HFM we first solve the eigenvalue problem of $H$, that is

$$
\boldsymbol{H} \boldsymbol{u}_{i}=\lambda_{i} \boldsymbol{u}_{i}, 1 \leq i \leq N
$$

where $\lambda_{i}$ is the $i$ th eigenvalue of $H$, and $\boldsymbol{u}_{i}$ is the eigenvector associated with $\lambda_{i}$. It is well known that all the eigenvalues of $H$ are real, since $H$ is Hermitian. It is also well known that all these eigenvectors are unitary, that is, mutually orthogonal in a complex space. Moreover, without loss of generality we can set $\lambda_{i} \neq 0(1 \leq i \leq n)$, and $\lambda_{n+1}=\lambda_{n+2}=\cdots=\lambda_{N}=0$. Then, let us define

$$
\boldsymbol{U}=\left[\boldsymbol{u}_{1}, \cdots, \boldsymbol{u}_{n}, \boldsymbol{u}_{n+1}, \cdots, \boldsymbol{u}_{N}\right]=\left[\boldsymbol{U}_{1}, \boldsymbol{U}_{2}\right],
$$

where $\boldsymbol{U}_{1}$ and $\boldsymbol{U}_{2}$ are composed of $n$ column vectors, $\boldsymbol{u}_{1}, \cdots, \boldsymbol{u}_{n}$, and $N-n$ column vectors, $\boldsymbol{u}_{n+1}, \cdots, \boldsymbol{u}_{N}$, respectively. Moreover, let us define

$$
\Lambda=\operatorname{diag}\left[\lambda_{1}, \lambda_{2}, \cdots, \lambda_{n}\right] .
$$

Then, using $\boldsymbol{U}_{1}$ and $\Lambda$, we get

$$
\boldsymbol{H}=\boldsymbol{U}_{1} \Lambda \boldsymbol{U}_{1}^{*} .
$$

Here, $\boldsymbol{U}_{1}^{*}$ denotes the conjugate transpose of $\boldsymbol{U}_{1}$.

Now, let us further define

$$
\Omega_{s}=\left[\begin{array}{cc}
\Lambda & O \\
O & \Lambda
\end{array}\right], \Omega_{s k}=\left[\begin{array}{cc}
O & -\Lambda \\
\Lambda & O
\end{array}\right]
$$

and

$$
\boldsymbol{X}=\left[\boldsymbol{U}_{r}, \boldsymbol{U}_{c}\right],
$$

where $\boldsymbol{U}_{r}=\left[r_{j l}\right]$ and $\boldsymbol{U}_{c}=\left[c_{j l}\right]$ are such that $\boldsymbol{U}_{1}=\boldsymbol{U}_{r}+i \boldsymbol{U}_{c}$. Here, $\boldsymbol{U}_{r}$ and $\boldsymbol{U}_{c}$ are, respectively, the real part and the imaginary part of the eigenvector matrix $U_{1}$ of Equation (6). Then, $\boldsymbol{H}$ in Equation (6) can be rewritten as

$$
\boldsymbol{H}=\boldsymbol{X} \boldsymbol{\Omega}_{\mathrm{s}} \boldsymbol{X}^{t}+i \boldsymbol{X} \boldsymbol{\Omega}_{\mathrm{sk}} \boldsymbol{X}^{t} .
$$

Here, it should be noticed that the matrix $\boldsymbol{X}, \boldsymbol{\Omega}_{s}$, and $\boldsymbol{\Omega}_{\mathrm{sk}}$ are all real. In scalar notation Equation (9) can be rewritten as

$$
h_{j k}=\sum_{l=1}^{n} \lambda_{l}\left\{\left(r_{j l} r_{k l}+c_{j l} c_{k l}\right)+i\left(c_{j l} r_{k l}-r_{j l} c_{k l}\right)\right\} .
$$


The $h_{j k}$ can be simply written, using Euler's formulas, as

$$
h_{j k}=\sum_{l=1}^{n} \lambda_{l} \eta_{l} \mathrm{e}^{i \theta_{l}}=G(\theta),
$$

where $\boldsymbol{\theta}=\left(\theta_{1}, \theta_{2}, \cdots, \theta_{n}\right)$.

Then, it is easy to see that $h_{j k}$ in Equation (11) has Hermitian symmetry, since the following equation holds:

$$
h_{j k}^{*}=G^{*}(-\theta) \text {, }
$$

and since $h_{j k}^{*}=\bar{h}_{k j}=h_{j k}$.

From Equation (9) we have the following equation

$$
\boldsymbol{S}=\boldsymbol{X} \boldsymbol{\Omega}_{\mathrm{s}} \boldsymbol{X}^{t}+\boldsymbol{X} \boldsymbol{\Omega}_{s k} \boldsymbol{X}^{t},
$$

using Equations (1) and (2). It should be noticed that all the elements in Equation (13) are real matrices. Equation (13) can be rewritten, in scalar notation, as

$$
s_{j k}=\sum_{l=1}^{n} \lambda_{l}\left\{\left(r_{j l} r_{k l}+c_{j l} c_{k l}\right)+\left(c_{j l} r_{k l}-r_{j l} c_{k l}\right)\right\} .
$$

In any case, if we write the $(j, k)$ element of $H$ in Equation (6) as $h_{j k}$, this equation can be rewritten in the form

$$
h_{j k}=\psi\left(v_{j}, v_{k}\right),
$$

where $\psi$ is a Hermitian form, $\psi\left(\boldsymbol{v}_{j}, \boldsymbol{v}_{k}\right)=\boldsymbol{v}_{j} \Lambda \boldsymbol{v}_{k}^{*}$, and the $n$-dimensional vectors, $\boldsymbol{v}_{j}$ and $\boldsymbol{v}_{k}$, are the $j$-th and $k$-th row vectors of $\boldsymbol{U}_{1}$, respectively. This is the reason why we call our model the Hermitian form model (HFM) for the analysis of asymmetry. Reference [18] proved that a necessary and sufficient condition for this model to be expressible in terms of Hilbert space is the positive semi-definiteness of $H$. If we use the bra-ket notation in quantum physics, the $\psi$ defined above can be expressed as

$$
\psi\left(v_{j}, v_{k}\right)=\left\langle v_{j}|\Lambda| v_{k}\right\rangle .
$$

In any case, if some of the eigenvalues of $H$ is negative, we can embed objects in an indefinite metric space. However, if we restrict the dimension into one in the indefinite metric space, this space can be considered as a one-dimensional Hilbert space, and we can interpret the configuration of objects as if it were embedded in a Hilbert space even if the eigenvalue under consideration is negative. In this case, however, we must interpret the configuration of objects, noticing the sign of the eigenvalue. We shall show such an example in Section 3.

Finally, we shall show an interesting identity in HFM. Let $\psi(\varsigma, \tau)$ be a Hermitian form. Then, we have the following polar identity in the (complex) pre-Hilbert space [20],

$$
\psi(\varsigma, \tau)=\frac{1}{4}\left(\|\varsigma+\tau\|^{2}-\|\varsigma-\tau\|^{2}\right)+\frac{1}{4} i\left(\|\varsigma+i \tau\|^{2}-\|\varsigma-i \tau\|^{2}\right) .
$$

This equation also holds for the Hilbert space. Then, remembering (10), it is easy to show that

$$
h_{j k}=\frac{1}{2}\left(\left\|v_{j}\right\|^{2}+\left\|v_{k}\right\|^{2}-\left\|v_{j}-v_{k}\right\|^{2}\right)+\frac{1}{2} i\left(\left\|v_{j}\right\|^{2}+\left\|v_{k}\right\|^{2}-\left\|v_{j}-i v_{k}\right\|^{2}\right) \text {. }
$$

Remembering (2), we have finally,

$$
s_{j k}=\left\|\boldsymbol{v}_{j}\right\|^{2}+\left\|\boldsymbol{v}_{k}\right\|^{2}-\frac{1}{2}\left(\left\|\boldsymbol{v}_{j}-\boldsymbol{v}_{k}\right\|^{2}+\left\|\boldsymbol{v}_{j}-i \boldsymbol{v}_{k}\right\|^{2}\right),
$$

where $s_{j k}$ is the $(j, k)$ element of $H$. This equation directly maps the complex vectors $\boldsymbol{v}_{j}$ and $\boldsymbol{v}_{k}$ in the Hilbert space, which cannot be observed, onto the measured result, $s_{j k}$. In this sense, $v_{j}$ and $\boldsymbol{v}_{k}$ can be said to be observables in terms of quantum mechanics. 


\subsection{Interpretation of the Configuration of Objects Obtained by HFM}

In this section, we explain briefly how to interpret the configuration of objects embedded in a Hilbert space, which is obtained by applying HFM to any empirical or theoretical ASM, according to [19]. As discussed in the previous section, objects are embedded in either the Hilbert space or the indefinite metric space depending on the eigenvalues of the Hermitian matrix $H$ constructed from the ASM, $S$. Here, it should be noticed that the $n$-dimensional row vectors, $\boldsymbol{v}_{j}$ and $\boldsymbol{v}_{k}$, in Equation (15) are coordinates of objects, $j$ and $k$, respectively, in the Hilbert space or the indefinite metric space. However, as also discussed in the previous section, both these spaces are considered as one-dimensional Hilbert spaces if we restrict the space to one-dimension. This means that we may think of the similarity, $s_{j k}$, in Equation (11) as

$$
s_{j k}=\lambda_{l}\left\{\left(r_{j l} r_{k l}+c_{j l} c_{k l}\right)+\left(c_{j l} r_{k l}-r_{j l} c_{k l}\right)\right\},
$$

For the $I$ th complex plane associated with the $t$ th eigenvalue of $\boldsymbol{H}$. Here, $r_{j l}$ and $c_{j l}$ are, respectively, the real part and the imaginary part of the complex number, $v_{j}$, corresponding to the location of object $\mathrm{j}$ (hereafter, abbreviate it as $O_{j}$ ), on the 1 th complex plane. Since the complex plane can be identified with the two-dimensional Euclidean plane, we can also consider $r_{j l}$ and $c_{j l}$, respectively, as the abscissa and the ordinate of the position of $O_{j}$, on the 1 th Euclidean plane.

Then, let us rewrite $r_{j l}$ and $c_{j l}$ as $x_{j 1}$ and $x_{j 2}$, respectively. Moreover, let us define the position vectors, $\boldsymbol{x}_{j}=\left[x_{j 1}, x_{j 2}\right]$ and $\boldsymbol{x}_{k}=\left[x_{k 1}, x_{k 2}\right]$ of $O_{j}$ and $O_{k}$, respectively. Then, Equation (20) can be rewritten as follows, if we introduce trigonometric functions:

$$
s_{j k}=\lambda\left|\boldsymbol{x}_{j}\right|\left|\boldsymbol{x}_{k}\right|\left(\cos \left(\theta_{j k}\right)-\sin \left(\theta_{j k}\right)\right),
$$

where $\theta_{j k}$ is the angle from $O_{j}$ and $O_{k}$. If we identify, respectively, real vectors, $\boldsymbol{x}_{j}$ and $\boldsymbol{x}_{k}$, with complex numbers, $v_{j}=x_{j 1}+i x_{j 2}$ and $v_{k}=x_{k 1}+i x_{k 2}, \theta_{j k}$ is the difference in the arguments $\theta_{j}$ and $\theta_{k}$ of $v_{j}$ and $v_{k}$, respectively, in a complex plane. Here, the argument of the complex number $z$, defined for $z \neq 0$, is the angle which the vector originating from 0 to $z$ makes with the positive real axis counterclockwise. Figure 1 illustrates $v_{j}$ and $v_{k}$ in a complex plane, assuming that $v_{j}$ is on the real axis. In this case, the argument of $v_{j}$ happens to be zero, and thus $\theta_{j k}$ is equal to the argument of $v_{k}$.

However, in interpreting the configuration of objects obtained by HFM, it should be noticed that the positive direction of the configuration of objects must be measured clockwise, considering Equations (20) and (21). This means that the similarity from $O_{k}$ associated with $v_{k}$ to $O_{j}$ associated with $v_{j}$ is relatively greater than that from $O_{j}$ to $O_{k}$ in Figure 1.

Remember here that the positive direction of the configuration of objects is counterclockwise in Chino's ASYMSCAL [21], which is one of the asymmetric MDSs. This model is a Euclidean space model and embeds objects in the two-dimensional Euclidean space. The original model is written as

$$
s_{j k}=a\left(x_{j 1} x_{k 1}+x_{j 2} x_{k 2}\right)+b\left(x_{j 1} x_{k 2}-x_{j 2} x_{k 1}\right)+c,
$$

where $a, b$, and $c$ are real constants.

If we identify real coordinate vector $x_{j}=\left[x_{j 1}, x_{j 2}\right]$ with the complex number $z_{j}=r_{j 1}+i c_{j 1}$ and $\boldsymbol{x}_{k}=\left[x_{k 1}, x_{k 2}\right]$ with the complex number $z_{k}=r_{k 1}+i c_{k 1}$, Equation (22) can be rewritten as

$$
s_{j k}=a\left(r_{j 1} r_{k 1}+r_{j 2} r_{k 2}\right)+b\left(r_{j 1} c_{k 2}-c_{j 2} r_{k 1}\right)+c .
$$

Now, let us set $a=b=\lambda$. Then, Equation (23) is written as

$$
s_{j k}=\lambda\left(r_{j 1} r_{k 1}+r_{j 2} r_{k 2}\right)+\lambda\left(r_{j 1} c_{k 2}-c_{j 2} r_{k 1}\right)+c .
$$

If we further rewrite Equation (21) as

$$
s_{j k}=\lambda\left(r_{j 1} r_{k 1}+r_{j 2} r_{k 2}\right)-\lambda\left(c_{j 2} r_{k 1}-r_{j 1} c_{k 2}\right)+c,
$$

and compare it with Equation (20) of HFM, we see that the positive direction of the configuration of 


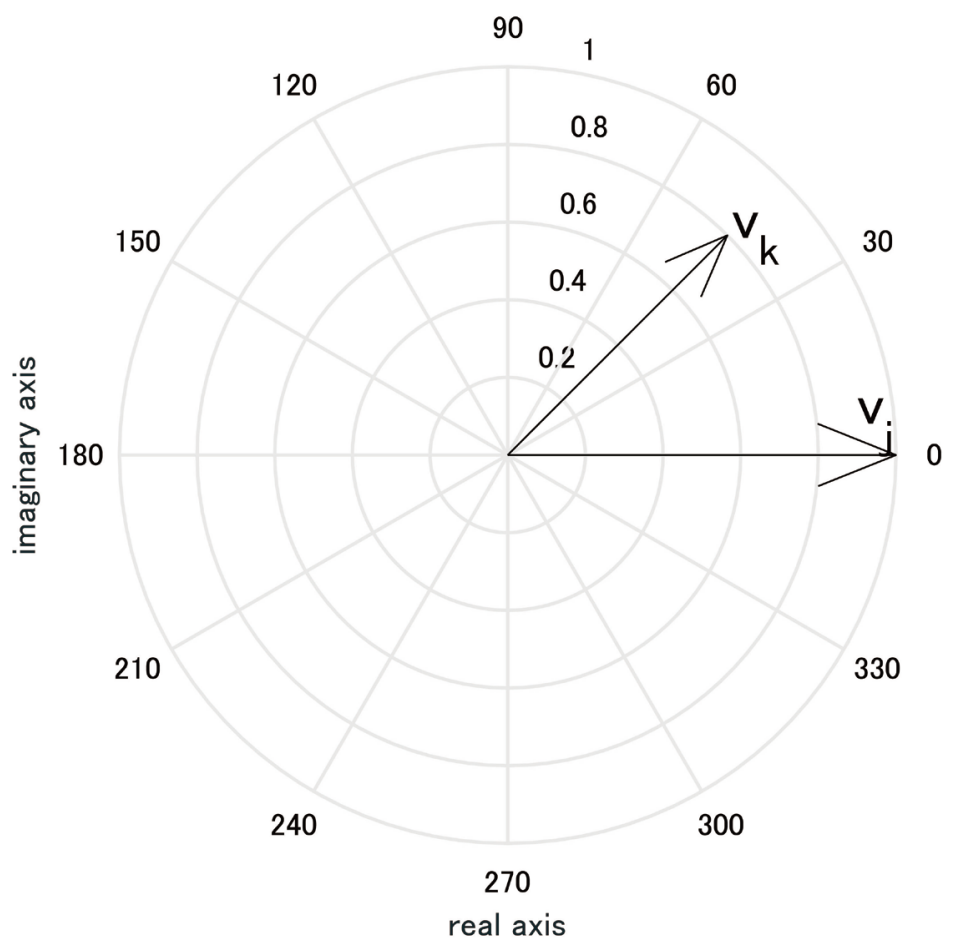

Figure 1. Configuration of two objects, $O_{j}$ and $O_{k}$ in the complex plane, and the argument $\theta_{j k}$ measured counterclockwise from $O_{j}$.

objects is opposite in the case of Chino's ASYMSCAL.

In any case, $s_{j k}$ in Equation (21) of HFM depends on $\theta_{j k}$ discussed there. Figure 2 and Figure 3 illustrate the details of $s_{j k}$ as a function of $\theta_{j k}$. In these figures, we set $\left|\boldsymbol{x}_{j}\right|\left|\boldsymbol{x}_{k}\right|$ in Equation (21) equal to 1 for simplicity. Moreover, we set $\lambda$ equal to 1 and -1 for Figure 2 and Figure 3, respectively. These correspond to the cases when the eigenvalues of $H$ are positive and negative, respectively.

It is apparent from Figure 2 that $s_{k j}$ is greater than $s_{j k}$ within the range, $0<\theta_{j k}<\pi$, while $s_{j k}$ is greater than $s_{k j}$ within the range, $\pi<\theta_{j k}<2 \pi$. Moreover, signs of $s_{j k}$ and $s_{k j}$ depend on $\theta_{j k}$. For more details, see elsewhere [19]. In contrast, it is apparent from Figure 3 that $s_{j k}$ is greater than $s_{k j}$ within the range, $0<\theta_{j k}<\pi$, while $s_{k j}$ is greater than $s_{j k}$ within the range, $\pi<\theta_{j k}<2 \pi$.

Finally, we shall consider a bit about the skewness between $s_{j k}$ and $s_{k j}$. It is easy to show that from Equation (21) we have

$$
s_{j k}-s_{k j}=-\lambda\left|\boldsymbol{x}_{j}\right|\left|\boldsymbol{x}_{k}\right| \sin \left(\theta_{j k}\right) .
$$

Therefore, if we set $\lambda\left|\boldsymbol{x}_{j}\right|\left|\boldsymbol{x}_{k}\right|$ equal to 1 , we have

$$
s_{j k}-s_{k j}=-\sin \left(\theta_{j k}\right) \text {, }
$$

and

$$
\left|s_{j k}-s_{k j}\right|=\left|\sin \left(\theta_{j k}\right)\right| \text {. }
$$

The green curve in Figure 4 is the very amount of the skewness between the similarities, $s_{j k}$ and $s_{k j}$.

From this figure, it is apparent that the skewness between them takes the maximum values when $\theta_{j k}$ is $\frac{\pi}{2}$ or $3 \frac{\pi}{2}$. 


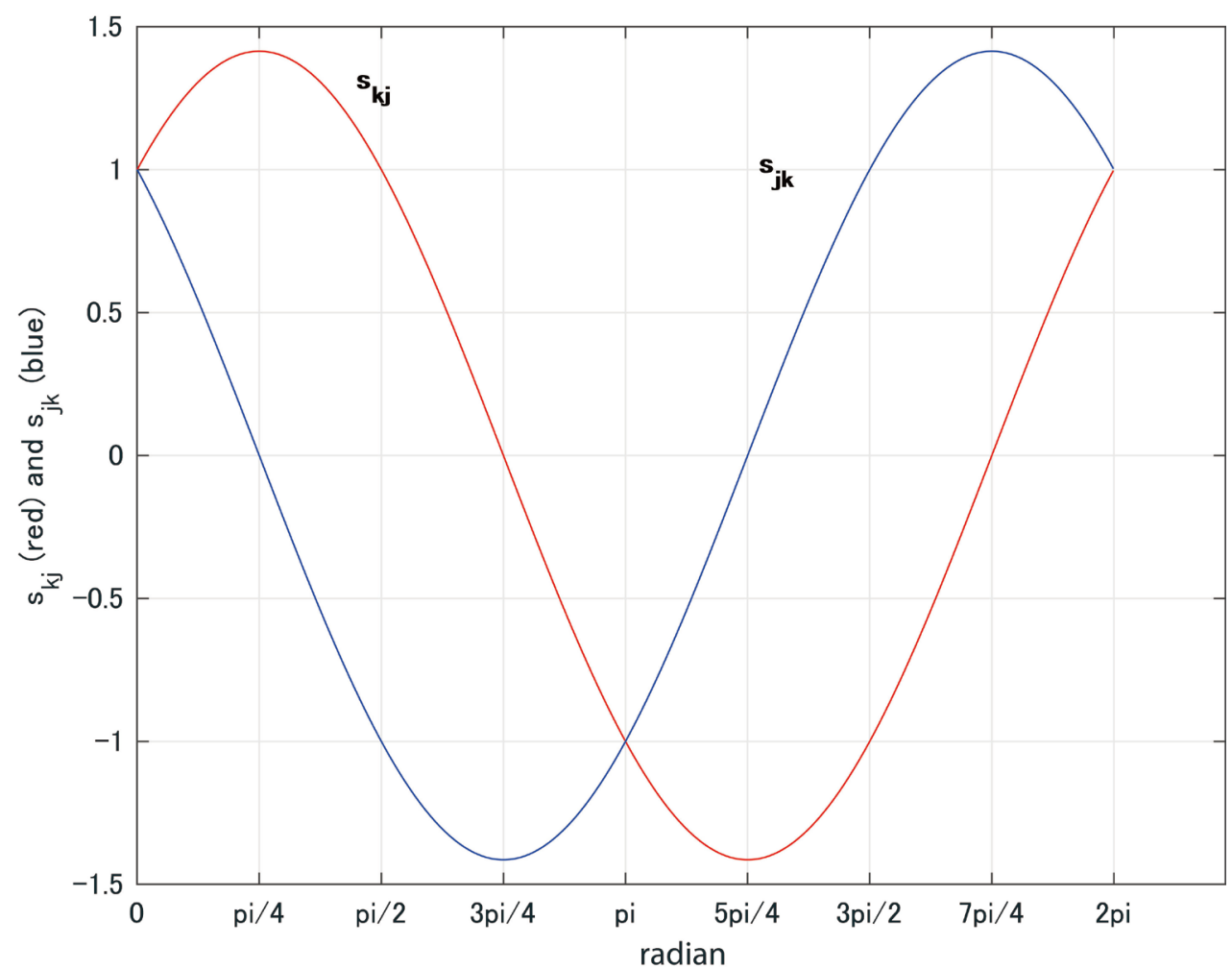

Figure 2. Values of $s_{j k}$ (blue curve) and $s_{k j}$ (red curve) plotted against $\theta_{j k}$ when the eigenvalue of $H$ is positive.

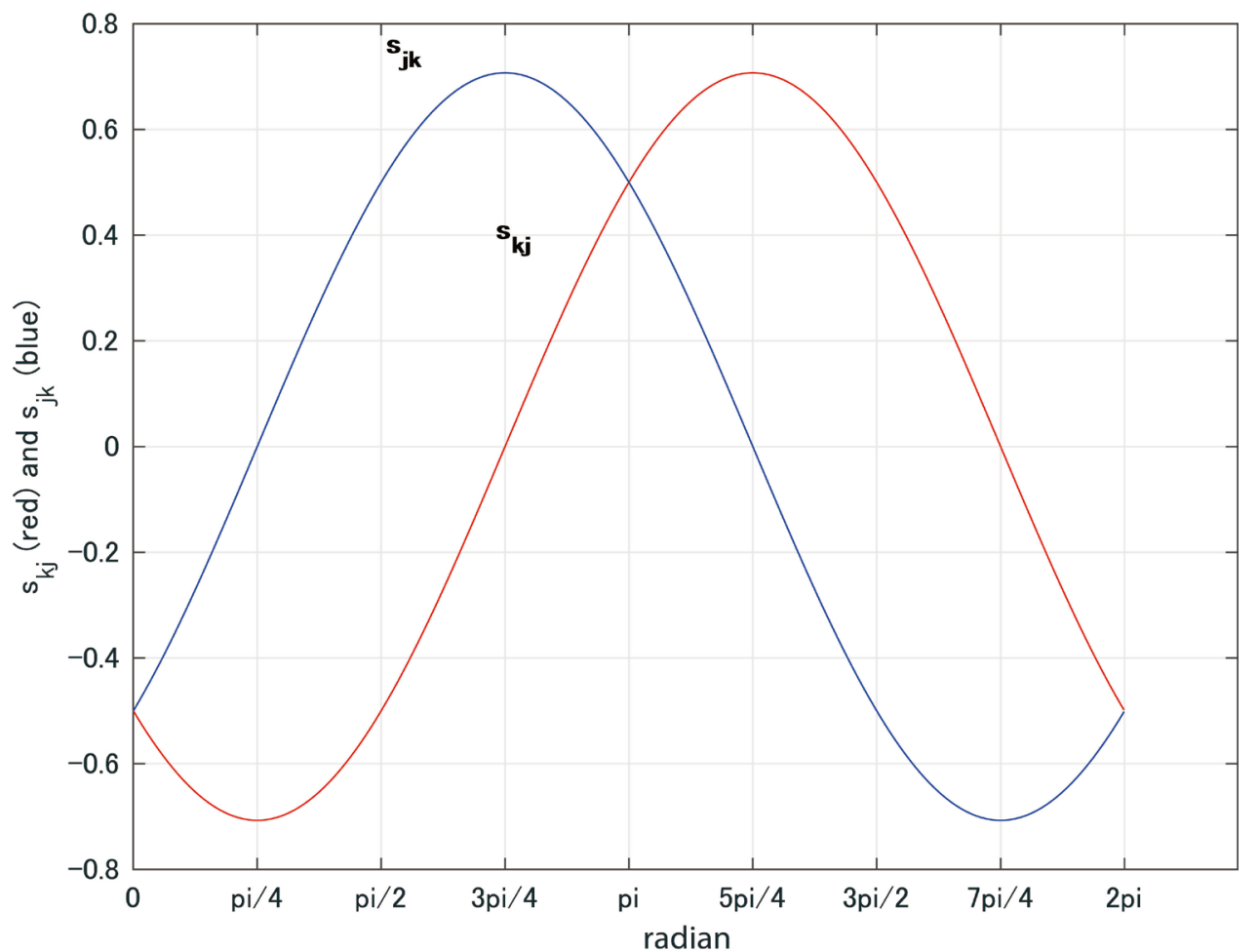

Figure 3. Values of $s_{j k}$ (blue curve) and $s_{k j}$ (red curve) plotted against $\theta_{j k}$ when the eigenvalue of $H$ is negative. 


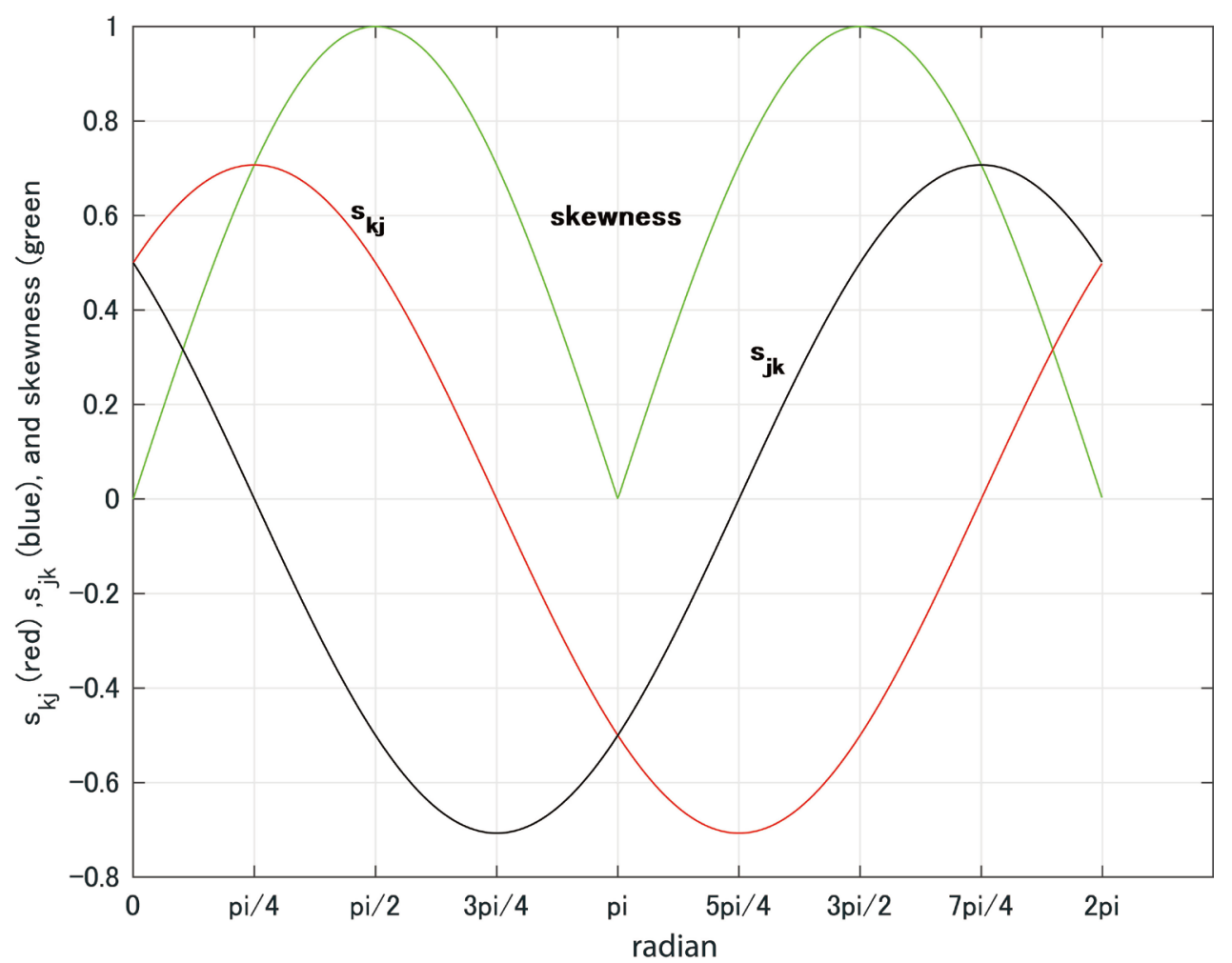

Figure 4. Skewness curves (green) between $s_{j k}$ (black curve) and $s_{k j}$ (red curve) plotted against $\theta_{j k}$.

\section{SIMILARITIES AND DIFFERENCES IN HILBERT SPACE USED IN QUANTUM MECHANICS AND PSYCHOMETRICS, ESPECIALLY IN HFM}

As discussed in the introduction, the density matrix operator $\hat{\boldsymbol{O}}$ of a wavefunction in quantum mechanics has the property of the positive semi-definite (p.s.d.) Hermitian matrix. In contrast, the complex matrix $H$ constructed from any real ASM between $N$ objects in psychometrics, especially in HFM, is a Hermitian matrix. Moreover, in HFM, these objects can be embedded in the Hilbert space if and only if $H$ is p.s.d. by the Chino and Shiraiwa theorem [18]. The similarities and differences of the Hermitian matrix in these two branches of sciences might be clearer, if we transform these two matrices, $\hat{\boldsymbol{O}}$ and $H$, in terms of usual matrix algebra.

As for the $\hat{\boldsymbol{O}}$, it is defined as follows, using the bra-ket notation,

$$
\hat{\boldsymbol{O}}=\sum_{k} \rho_{k}\left|\psi_{k}\right\rangle\left\langle\psi_{k}\right|,
$$

as discussed in the introduction section. Since $\left|\psi_{k}\right\rangle$ is the $k$ th eigenvector (called sometimes eigenkets in quantum mechanics) associated with the $k$ th eigenvalue of $\hat{\boldsymbol{O}}, \hat{\boldsymbol{O}}$ is expressed, in terms of the usual matrix algebra, as

$$
\hat{\boldsymbol{O}}=\sum_{k} \rho_{k} \boldsymbol{\psi}_{k} \boldsymbol{\psi}_{k}^{*}=\boldsymbol{V D} \boldsymbol{V}^{*},
$$

where $\boldsymbol{V}=\left[\boldsymbol{\psi}_{1}, \boldsymbol{\psi}_{2}, \cdots, \boldsymbol{\psi}_{M}\right]$, and $\boldsymbol{D}=\operatorname{diag}\left[\rho_{1}, \rho_{2}, \cdots, \rho_{M}\right]$, and $\boldsymbol{\psi}_{k}^{*}$ and $\boldsymbol{V}^{*}$ are the conjugate transposes of $\psi_{k}$ and $V$, respectively. Equation (30) can be expressed, using the row vectors, $\varphi_{1}, \varphi_{2}, \cdots, \varphi_{p}$ composed of the rows of $V$, as

$$
\hat{\boldsymbol{O}}=\left[\hat{\rho}_{i j}\right]=\left[\boldsymbol{\varphi}_{i} \boldsymbol{D} \boldsymbol{\varphi}_{j}^{*}\right] .
$$

Since $\hat{\rho}_{i j}$ is a Hermitian form, and since $\hat{\boldsymbol{O}}$ is p.s.d., $\hat{\rho}_{i j}$ is the Hermitian inner product. 
As regards the $H$, we have Equation (6), which is nothing but the direct result of the eigenvalueeigenvector decomposition of $H$. This equation corresponds to Equation (30) in quantum mechanics.

The Hermitian form derived from $H$ is Equation (15). This equation corresponds to Equation (31) in

Quantum mechanics. Both Hermitian forms, $h_{j k}=\psi\left(\boldsymbol{v}_{j}, \boldsymbol{v}_{k}\right)=\boldsymbol{v}_{j} \boldsymbol{\Lambda} \boldsymbol{v}_{k}^{*}$ and $\hat{\rho}_{i j}=\boldsymbol{\varphi}_{i} \boldsymbol{D} \boldsymbol{\varphi}_{j}^{*}$, are Hermitian symmetric, i.e., $h_{j k}=\overline{h_{j k}}$, and $\hat{\rho}_{i j}=\overline{\hat{\rho}}_{i j}$. The notion of Hermitian symmetry is an extension of that of usual symmetry to complex number. Under a mild condition, i.e., p.s.d.'s, of $\hat{\boldsymbol{O}}$ as well as $H$, guarantee that $\varphi_{1}, \varphi_{2}, \cdots$ as well as $v_{1}, v_{2}, \cdots$ are embedded in a Hilbert space. Therefore, Hermitian symmetry is a fundamental principle in the microscopic world, i.e., in quantum mechanics, as well as in the medium-size world in which real ASM $s$ are observed or hypothesized, examples of which are neural networks, biological networks, social networks, and so on.

\section{APPLICATIONS OF HFM TO SOME ASYMMETRIC PHENOMENA}

In this section we shall apply HFM to some empirical ASMs and a hypothetical ASM. The first example is concerned with the biosynthetic pathways of proteins in a budding yeast and mammals (e.g. [1]), which appears elsewhere [19]. We chose an example of which appears in Fig.2b of [1]. We reconstruct an ASM from this figure in such a way that the similarity is 1 if the directional arc exists from an element to another element of 11 substances, and otherwise it is 0 . These substances are composed of tryptophan (an $\alpha$-amino acid), NAD (nicotinamide adenine dinucleotide, a non-protein chemical compound), NIC (nicotinamide, an organic molecule), and so on. Table 1 shows this. Here, it should be noticed that in general such a pathway can be considered as a weighted digraph (directed graph) in graph theory. Since the weighted digraph accompanies a weight matrix, we say that in general a unique ASM is associated with any weighted digraph.

Eigenvalues of the Hermitian matrix constructed from this ASM were $1.6302,-1.6302,-1.2534$, $1.2534,-0.8706,0.8706, \ldots$ These eigenvalues mean that this data has a holistic indefinite metric structure. Of course, each of the configurations of the biosynthetic pathways of proteins in mammals on the complex planes associated with these eigenvalues can be embedded in a one-dimensional Hilbert space, as discussed previously.

Table 1. A biosynthetic pathway of proteins in mammals which was re-constructed from Figure 2 of [1].

\begin{tabular}{|c|c|c|c|c|c|c|c|c|c|c|c|}
\hline & 1 & 2 & 3 & 4 & 5 & 6 & 7 & 8 & 9 & 10 & 11 \\
\hline 1 & 0 & 1 & 0 & 0 & 0 & 0 & 0 & 0 & 0 & 0 & 0 \\
\hline 2 & 0 & 0 & 0 & 1 & 0 & 0 & 0 & 0 & 0 & 0 & 0 \\
\hline 3 & 0 & 0 & 0 & 1 & 0 & 0 & 0 & 0 & 0 & 0 & 0 \\
\hline 4 & 0 & 0 & 0 & 0 & 1 & 0 & 0 & 0 & 0 & 0 & 0 \\
\hline 5 & 0 & 0 & 0 & 0 & 0 & 1 & 0 & 0 & 0 & 0 & 0 \\
\hline 6 & 0 & 0 & 0 & 0 & 0 & 0 & 1 & 0 & 1 & 0 & 0 \\
\hline 7 & 0 & 0 & 0 & 0 & 0 & 0 & 0 & 0 & 0 & 0 & 0 \\
\hline 8 & 0 & 0 & 0 & 0 & 0 & 0 & 0 & 0 & 0 & 0 & 0 \\
\hline 9 & 0 & 0 & 0 & 0 & 0 & 0 & 0 & 1 & 0 & 0 & 0 \\
\hline 10 & 0 & 0 & 0 & 0 & 0 & 1 & 0 & 0 & 0 & 0 & 1 \\
\hline 11 & 0 & 0 & 0 & 0 & 0 & 0 & 0 & 0 & 0 & 1 & 0 \\
\hline
\end{tabular}


Figure 5 shows four configurations of the biosynthetic pathways composed of these one-dimensional Hilbert spaces. These four configurations are associated with the first four eigenvalues of the Hermitian matrix discussed above.

Figure 5(a) is the configuration associated with the maximum eigenvalue of the Hermitian matrix. Although this configuration recovers the major pathways contained in the original data matrix, it does not include the important cyclic pathways, starting from $O_{6}(\mathrm{NAD})$ to come back to it through $O_{9}$ (NIC) and $O_{10}(\mathrm{NMN})$. In contrast, Figure 5(b), which is the configuration corresponding to the maximum eigenvalue but with a negative sign, recovers almost all the pathways contained in the original matrix. Figure 6 is an enlarged configuration of Figure 5(b). In interpreting this configuration, it should be noticed that its positive direction is counterclockwise because the eigenvalue associated with it is negative. Noticing this point, we can find the following pathways in Figure 6 by following an object to another object counterclockwise within $\pi$ radian, referring to Figure 2 of [1]:

1) $O_{1}$ (Tryptophan) $\rightarrow O_{2}$ (Quinolinic acid) $\rightarrow O_{4}(\mathrm{NaMN}) \rightarrow O_{5}\left(\right.$ deamido-NAD) $\rightarrow O_{6}(\mathrm{NAD}) \rightarrow O_{9}$ $(\mathrm{NIC}) \rightarrow O_{10}(\mathrm{NMN}) \rightarrow O_{6}(\mathrm{NAD})$

2) $O_{3}(\mathrm{NA}) \rightarrow O_{4}(\mathrm{NaMN})$

The first pathway includes a cycle starting from $O_{6}$ and returning to itself. The second pathway runs into the first pathway at $O_{4}(\mathrm{NaMN})$. The third pathway gets away from the first pathway at $O_{9}$ (NIC). The fourth pathway also gets away from the first pathway at $\mathrm{O}_{6}$ (NAD).

The second example is the international trade data among Japan, America, China, and Russia in 2015, which appeared in The Asahi News Paper in Japan, which is discussed elsewhere [19]. Table 2 shows this. According to convention, we administered a log transformation to each element of this ASM prior to the analysis via HFM. Eigenvalues of the Hermitian matrix constructed from the transformed ASM were 29.9714, 61145, 4.4377, and 3.5309. This means that the trade data has a holistic Hilbert space structure. Of course, each of the configurations of nations on the complex planes associated with these eigenvalues is

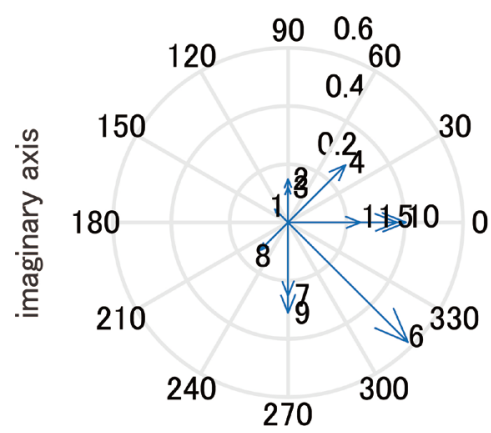

(a) Coordinates in dimension 1

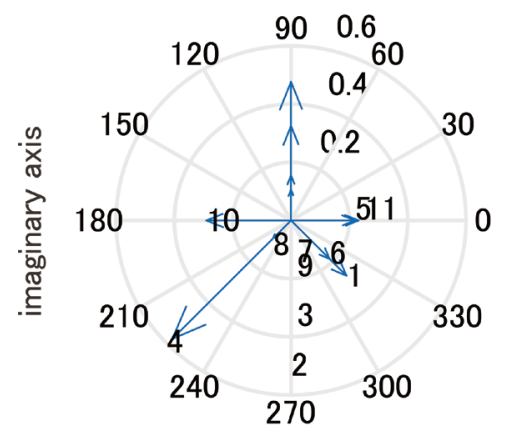

(c) Coordinates in dimension 3

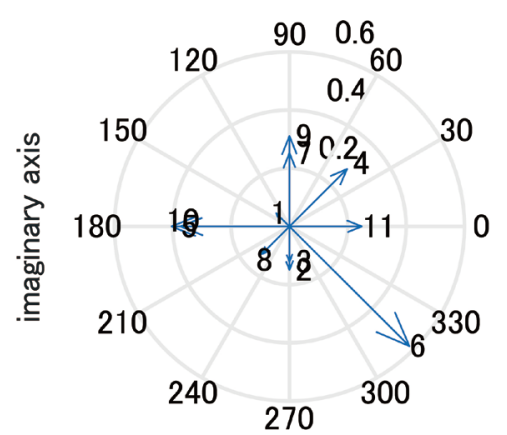

(b) Coordinates in dimension 2

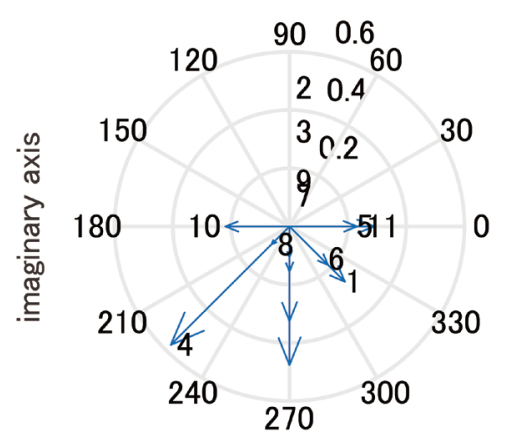

(d) Coordinates in dimension 4

Figure 5. Four configurations of the biosynthetic pathways corresponding to one-dimensional Hilbert spaces, which constitute a part of the holistic 11-dimensional Hilbert space. 


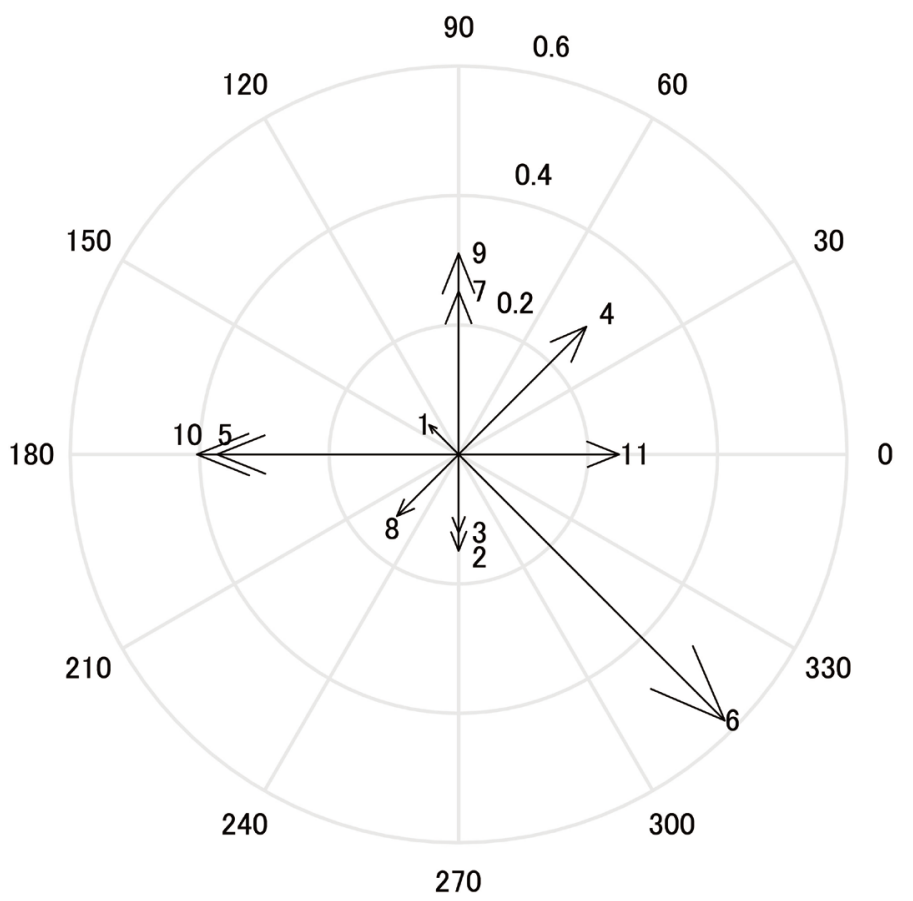

Figure 6. An enlarged configuration of Figure 4(b).

Table 2. The international trade data among Japan, America, China, and Russia in 2015.

\begin{tabular}{lcccc}
\hline & 1. Japan & 2. USA & 3. China & 4. Russia \\
\hline 1. Japan & 43,480 & 1382 & 1200 & 55 \\
2. USA & 736 & 189,592 & 1161 & 71 \\
3. China & 1764 & 4832 & 119,684 & 348 \\
4. Russia & 173 & 164 & 333 & 13,755 \\
\hline
\end{tabular}

considered as embedded in a one-dimensional Hilbert space, as indicated in the previous section. Moreover, these configurations embedded in one-dimensional Hilbert spaces are mutually orthogonal (to be precise, unitary) in the whole space with the complex Hilbert space structure.

Figure 7 shows four configurations of nations corresponding to these one-dimensional Hilbert spaces. Of course, these four configurations are associated with the four eigenvalues of the Hermitian matrix discussed above.

Figure 7(a), which is the configuration associated with the maximum eigenvalue of the Hermitian matrix, suggests that this dimension looks like a size-factor in PCA because four nations are compressed in a narrow region. However, if we enlarge this region, we see that major asymmetric relationships among nations observed in the ASM are reproduced in the enlarged configuration. Here, in interpreting this configuration, it should be noticed that the positive direction of this figure is clockwise because the eigenvalue is positive in this case. It should also be noticed that angles $\theta_{j k}$ between two nations all fall within $\pi / 3$ (30 degree), as is apparent from Figure 7(a). Then, looking at Figure 2, we see that $s_{k j}>s_{j k}$. As a result, applying this relation to nations in Figure 8, the amount of export from C (China) to A (USA) is greater than that from A to C. Similar trade imbalances are indicated from C to J, and from J to A. Finally, it should be noticed that the magnitude of the skewness between $s_{j k}$ and $s_{k j}$, which is defined by Equation 


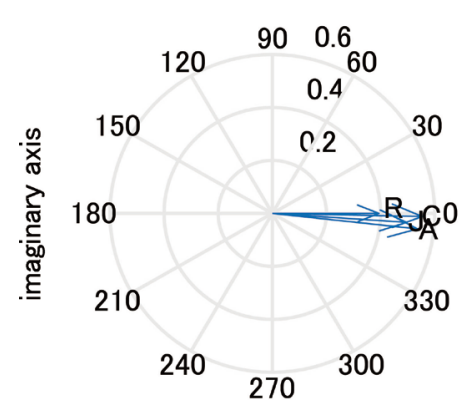

(a) Coordinates in dimension 1

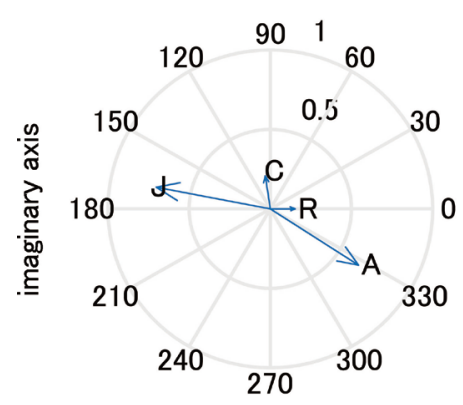

(c) Coordinates in dimension 3

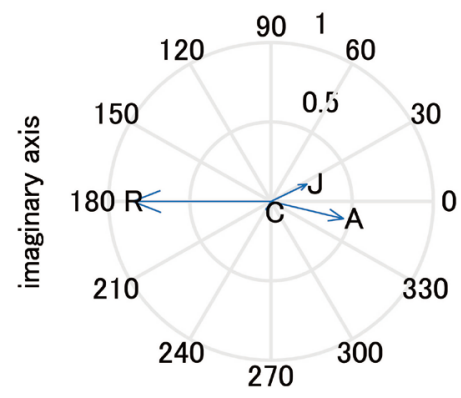

(b) Coordinates in dimension 2

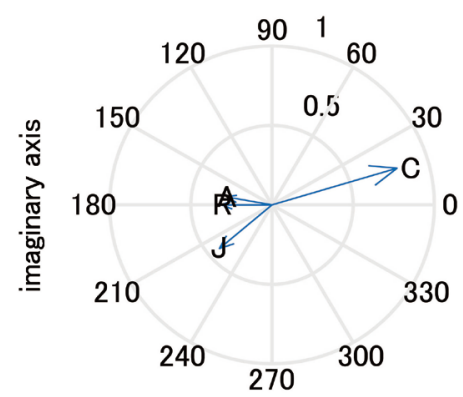

(d) Coordinates in dimension 4

Figure 7. Four configurations of nations corresponding to the one-dimensional Hilbert space. In this figure, alphabets, A, C, J, and R, indicate USA, China, Japan, and Russia.

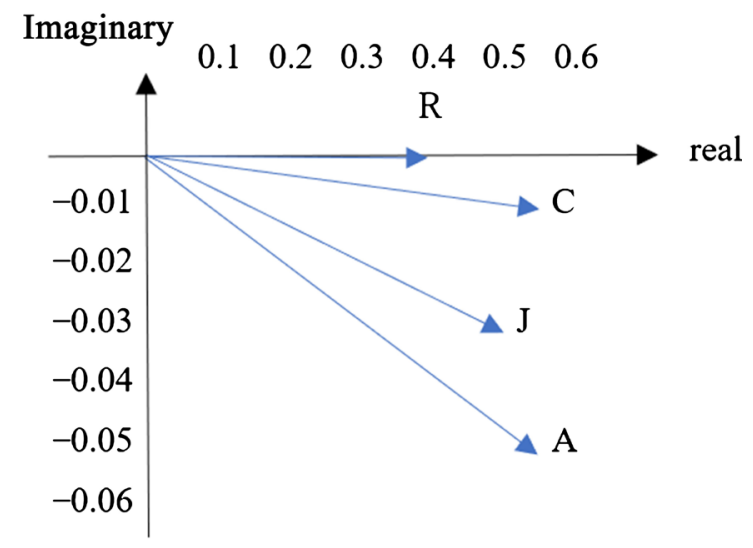

Figure 8. The enlarged configuration of four nations. Here, the positive direction of this figure is clockwise because the eigenvalue is positive in this case.

(25), increases as $\theta_{j k}$ increases within $\pi / 3$, as shown in Figure 4. Since this magnitude is also proportional to $\left|\boldsymbol{x}_{j}\right|\left|\boldsymbol{x}_{k}\right|$ in Equation (26), the magnitude of skewness between A and C might be the greatest of all the dyadic relations shown in Figure 8.

The third example is the application of HFM to theoretical or hypothetical asymmetric relational data matrices [19]. Reference [23] investigated the problem of learning to play the game of rock-paper-scissors, using the following set of nonlinear differential equations.

$$
\left[\begin{array}{c}
\dot{x}_{i} \\
\dot{y}_{j}
\end{array}\right]=\left[\begin{array}{c}
x_{i}(\boldsymbol{A y})_{i}-\boldsymbol{x}^{t} \boldsymbol{A} \boldsymbol{y} \\
y_{j}(\boldsymbol{B} \boldsymbol{x})_{j}-\boldsymbol{y}^{t} \boldsymbol{A} \boldsymbol{x}
\end{array}\right], \quad i=1, \cdots, n, \quad j=1, \cdots, m .
$$


where $\boldsymbol{x}=\left(x_{1}, \cdots, x_{n}\right)^{t}$ is the relative frequency vector for one population, while $\boldsymbol{y}=\left(y_{1}, \cdots, y_{m}\right)^{t}$ is that for the second population. This approach is based on the theory of games and the notion of evolutionarily stable strategy (abbreviated as ESS), of which theory was introduced by [24].

In any case, in Eq. (32) $A$ is the payoff matrix for one population, while $B$ is the payoff matrix for the second population, and these two matrices are denoted as

$$
\boldsymbol{A}=\left[\begin{array}{ccc}
\varepsilon_{x} & -1 & 1 \\
1 & \varepsilon_{x} & -1 \\
-1 & 1 & \varepsilon_{x}
\end{array}\right], \quad \boldsymbol{B}=\left[\begin{array}{ccc}
\varepsilon_{y} & -1 & 1 \\
1 & \varepsilon_{y} & -1 \\
-1 & 1 & \varepsilon_{y}
\end{array}\right],
$$

where $-1<\varepsilon_{x}<1$ and $-1<\varepsilon_{y}<1$. Here, columns of these matrices are placed in the order of "rock", "paper", and "scissors". If $\varepsilon_{x}=-\varepsilon_{y}=\varepsilon$, this game is called a zero sum game. In matrix notation, this condition is denoted as $\boldsymbol{A}=-\boldsymbol{B}^{t}$. Matrices, $\boldsymbol{A}$ and $\boldsymbol{B}$, are nothing but theoretical examples of ASM, in that these ASMs cannot be observed and are hypothesized a priori.

Although [23] discuss these matrices from the viewpoint of a dynamical system, they do not discuss the metric structure of these matrices. Such a structure can be examined by applying HFM to them. Let us now examine the structure of $\boldsymbol{A}$ in the case when $\varepsilon_{x}=0.25$, which is one of the cases in which $\varepsilon_{x}$ and $\varepsilon_{y}$ are treated as a bifurcation parameter of the dynamical system described by Equation (32). The eigenvalues of the Hermitian matrix constructed from this ASM were 1.9821, $-1.4821,0.2500$. These eigenvalues mean that this data has a holistic indefinite metric structure. Figure 9 shows this structure. Each of the configurations shows the positions of the three elements, i.e., "1. rock", "2. paper", and "3. scissors", in a one-dimensional Hilbert space. For further details, see [19].

\section{DISCUSSION}

In considering mutual interactions among objects in various disciplines of sciences, we usually discuss two aspects, one being the space in which objects interact with one another, and the other being the dynamics by which objects interact. In this paper, we have restricted our attention to the former and have had a

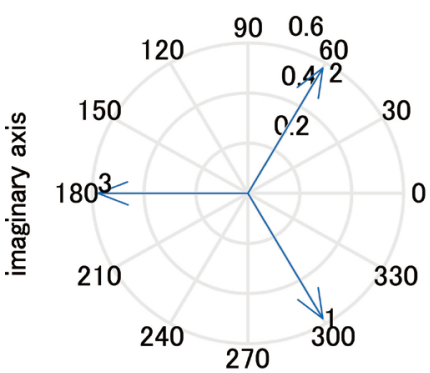

(a) Coordinates in dimension 1

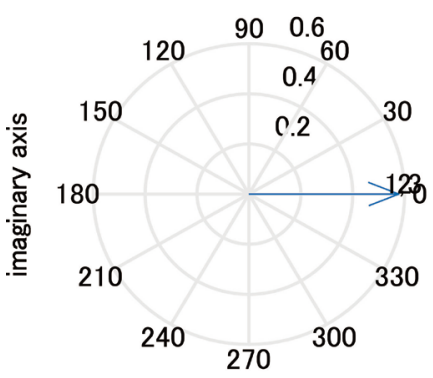

(c) Coordinates in dimension 3

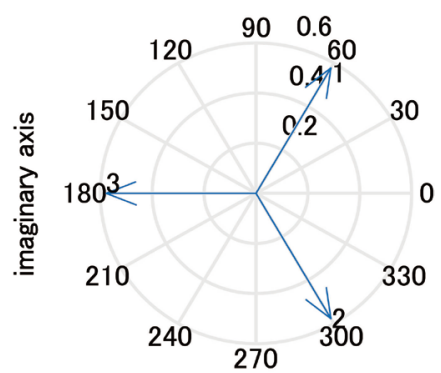

(b) Coordinates in dimension 2

Figure 9. Three configurations of the payoff matrix A corresponding to one-dimensional Hilbert spaces, which constitute the holistic 3-dimensional Hilbert space. 
glance at the various spaces in which objects are embedded, first. In the macroscopic world, symmetric metric spaces, i.e., the Minkowski metric space and the Riemannian metric space, are required according to the special and general relativity theories, respectively [15]. In the microscopic world, Hilbert spaces are necessary to discuss states of the elementary particles, which are complex spaces (unitary spaces) whose metric is induced by the Hermitian inner products (e.g., [20]). In modern quantum mechanics, PT symmetry, which is a generalization of the condition of Hermitian in Hamiltonian systems, has been discussed (e.g., [22]). However, if we look at the medium-sized world, interactions among objects, say, neurons or boxels of neurons, several proteins in a budding yeast, a few species of corals, members in a class, social classes, nations, and so on, are asymmetric in most cases. Then, what is the most appropriate metric space in which objects can be embedded, given the observed or hypothesized ASM whose elements denote certain similarities between objects? Here, these similarities must be measured or hypothesized at the ratio scale level, which is defined in psychometrics. Asymmetric MDS techniques developed in psychometrics, especially the HFM proposed by [18] has an answer to this question.

As revisited in Section 2, HFM transforms such an ASM, say, $\mathcal{S}$, into a Hermitian matrix, $H$, first. Then, HFM performs the eigenvalue-eigenvector decomposition of $H$, and embeds objects in a finite-dimensional Hilbert space or in an indefinite metric space, depending on whether $H$ is p.s.d. or not. In any case, as HFM transforms the observed or hypothesized similarity between objects into the Hermitian form, it naturally possesses the property of Hermitian symmetry in regard to the argument of the corresponding complex quantity, as was discussed in that section.

In Section 3, we have discussed the similarities and differences in the Hilbert space used in quantum mechanics and psychometrics, especially, in HFM. The Hermitian matrix is the density matrix operator in quantum mechanics, while it is the complex matrix constructed from the observed or hypothesized similarities among objects in HFM. If both Hermitian matrices are p.s.d., then we can embed the state of an elementary particle as well as objects in HFM into the finite-dimensional Hilbert space.

In Section 4, we have shown some applications of HFM to ASMs observed or hypothesized in some branches of science. Although we could not show applications to the other branches of sciences such as community ecology, there exist various examples accumulated in this area of research (e.g., [25, 26]), which are depicted as digraphs.

As regards the dynamics by which objects interact in the medium-sized world, we have been developing a nonlinear complex difference equation model whose state spaces are a finite-dimensional Hilbert space or an indefinite metric space (e.g., [27-29]). We shall publish a final version of this model elsewhere in the near future.

\section{ACKNOWLEDGEMENTS}

The author is indebted to Gregory L. Rohe for proofreading of this paper.

\section{CONFLICTS OF INTEREST}

The author declares no conflicts of interest regarding the publication of this paper.

\section{REFERENCES}

1. Imai, S. and Guarente, L. (2014) $\mathrm{NAD}^{+}$and Sirtuins in Aging and Disease. Trends in Cell Biology, 24, 464-471. https://doi.org/10.1016/j.tcb.2014.04.002

2. Parsek, M.R. and Greenberg, E.P. (2005) Sociomicrobiology: The Connections between Quorum Sensing and Biofilms. Trends in Microbiology, 13, 27-33. https://doi.org/10.1016/j.tim.2004.11.007

3. Sato, Y., Akiyama, E. and Farmer, J.D. (2002) Chaos in Learning a Simple Two-Person Game. Proceedings of the National Academy of Science of the United States of America, 99, 4748-4751. https://doi.org/10.1073/pnas.032086299 
4. Tian, J., Huang, R., Osakada, F., Kobak, D., Machens, C.K., Callaway, E.M., Uchida, H. and Watanabe, M. (2016) Distributed and Mixed Information in Monosynaptic Inputs to Dopamine Neurons. Neuron, 91, 1-16.

https://doi.org/10.1016/j.neuron.2016.08.018

5. Vega, N.M., Allison, K.R., Samuels, A.N., Klempner, M.S. and Collins, J.J. (2013) Salmonella Typhimurium Intercepts Escherichia Coli Signaling to Enhance Antibiotic Tolerance. Proceedings of the National Academy of Sciences, 110, 1442014425. https://doi.org/10.1073/pnas.1308085110

6. Yamada, H., Toth, A. and Nakagaki, T. (2000) Intelligence: Maze-Solving by an Amoeboid Organism. Nature, 407, 470. https://doi.org/10.1038/35035159

7. Bang-Jensen, J. and Guttin, G. (2007) Digraphs-Theory, Algorithms and Applications. Springer-Verlag, New York.

8. Christofides, N. (1975) Graph Theory-An Algorithmic Approach. Academic Press, New York.

9. Schultz, W., Dayan, P. and Montague, P.R. (1997) A Neural Substrate of Prediction and Reward. Science, 275, 1593-1599. https://doi.org/10.1126/science.275.5306.1593

10. Chino, N. (2012) A Brief Survey of Asymmetric MDS and Some Open Problems. Behaviormetrika, 39, 127-165. https://doi.org/10.2333/bhmk.39.127

11. Close, F. (2000) Lucifer's Legacy: The Meaning of Asymmetry. Oxford University Press, Oxford.

12. Chino, N. (1978) A Graphical Technique for Representing the Asymmetric Relationships between N Objects. Behaviormetrika, 5, 23-40. https://doi.org/10.2333/bhmk.5.23

13. Maynard, S. and Price, G.R. (1973) The Logic of Animal Conflict. Nature, 246, 15-18. https://doi.org/10.1038/246015a0

14. Wechsler, D. (1944) The Measurement of Adult Intelligence. 3rd Edition, The Williams \& Wilkins Company, Baltimore, MD.

15. Einstein, A. (1916) Die Grundlage der Allgemeinen Relativitätstheorie [The Foundation of the Generalized Theory of Relativity]. Annalen der Physik, 4, 284-339.

16. Born, M. (1926) Zur Quantenmechanik der Stoßvogänge. Zeitschrift für Physik, 37, 863-867. https://doi.org/10.1007/BF01397477

17. Stefanucci, G. and van Leeuwen, R. (2013) Nonequilibrium Many-Body Theory of Quantum Systems: A Modern Introduction. Cambridge University Press, Cambridge.

18. Chino, N. and Shiraiwa, K. (1993) Geometrical Structures of Some Non-Distance Models for Asymmetric MDS. Behaviormetrika, 20, 35-47. https://doi.org/10.2333/bhmk.20.35

19. Chino, N. (2020) How to Use the Hermitian Form Model for Asymmetric MDS. In: Imaizumi, T., Nakayama, A. and Yokoyama, S., Eds., Advanced Studies in Behaviometrics and Data Science, Springer, Tokyo. (In Print) https://doi.org/10.1007/978-981-15-2700-5 2

20. Cristescu, R. (1977) Topological Vector Spaces. Noordhoff International Publishing, Leyden.

21. Chino, N. (1978) A Graphical Technique for Representing the Asymmetric Relationships between N Objects. Behaviormetrika, 5, 23-40. https://doi.org/10.2333/bhmk.5.23

22. Bender, C.M. (2019) PT Symmetry in Quantum and Classical Physics. World Scientific, Singapore.

23. Sato, Y., Akiyama, E. and Farmer, J.D. (2002) Chaos in Learning a Simple Two-Person Game. Proceedings of the National Academy of Sciences of the United States of America, 99, 4748-4751. https://doi.org/10.1073/pnas.032086299

24. Maynard Smith, J. and Price, G.R. (1973) The Logic of Animal Conflict. Nature, 246, 15-18. https://doi.org/10.1038/246015a0

25. Chadwick-Furman, N. and Rinkevich, B. (1994) A Complex Allorecognition System in a Reef-Building Coral: Delayed Responses, Reversals and Nontransitive Hierarchies. Coral Reefs, 13, 57-63. https://doi.org/10.1007/BF00426436

26. Masure, R.H. and Allee, W.C. (1934) The Social Order in Flocks of the Common Chicken and the Pigeon. The Auk, 51, 306-327. https://doi.org/10.2307/4077659

27. Chino, N. (2002) Complex Difference System Models for the Analysis of Asymmetry. In: Yanai, H., Okada, A., Shigemasu, K., Kano, Y. and Meulman, J.J., Eds., New Developments in Psychometrics, Springer, Tokyo, 479-486. 
28. Chino, N. (2018) An Elementary Theory of a Dynamic Weighted Digraph (1). Bulletin of the Faculty of Psychological \& Physical Science, 14, 23-31.

29. Chino, N. (2018) Dynamical Scenarios of Changes in Asymmetric Relationships over Time (2). Journal of the Institute for Psychological and Physical Science, 10, 7-14. 